\title{
Identificação de Sistemas Empregando Técnica de Otimização Multiobjetivo para Seleção de Estrutura e Parâmetros de Modelo do tipo FBO-Volterra Aplicada à Modelagem de uma Câmara Termoelétrica
}

\author{
Alisson Marden F. Pereira ${ }^{*, * *}$ Víctor C. S. Costa ${ }^{* *}$ \\ Márcio F. Braga ${ }^{*, * * *}$ \\ * Departamento de Engenharia Elétrica, Instituto de Ciências Exatas e \\ Aplicadas, Universidade Federal de Ouro Preto - UFOP. \\ ** Departamento de Engenharia Eletrônica, Universidade Federal de \\ Minas Gerais - UFMG. \\ *** Instituto Tecnológico Vale, $M G-I T V$. \\ E-mails:alisson_fonseca2@yahoo.com.br,victor@cpdee.ufmg.br, \\ mfbraga@ufop.edu.br.
}

\begin{abstract}
This work aims to model a thermo-electrical system, using Volterra series in association with Orthonormal Basis Function (OBF). A multi-objective optimization algorithm is used to obtain the OBF optimum pole and to reduce the number of necessary parameters to the identification procedure. The algorithm employed is of the class of Multiobjective Evolutionary Algorithms (MOEA) and the system used to illustrate the technique applicability is a thermoelectrical chamber whose temperature is varied by Peltier modules.

Resumo: Este trabalho tem como objetivo fazer a modelagem de um sistema termoelétrico, usando as series de Volterra em conjunto com as Funções de Base Ortonormal (FBO). Neste trabalho, emprega-se otimização multiobjetivo para obter o polo ótimo da FBO e reduzir o número de parâmetros necessários para a identificação do modelo. O algoritmo utilizado é da classe dos Multiobjective Evolutionary Algorithms (MOEA) e o sistema utilizado para ilustrar a aplicação da técnica é uma câmara térmica com temperatura variada por meio de módulos Peltier.
\end{abstract}

Keywords: Systems identification, OBF-Volterra Models, Multiobjective optimization, Multiobjective Evolutionary Algorithms (MOEA), Thermoelectric Chamber.

Palavras-chaves: Identificação de sistemas, Modelos FBO-Volterra, Otimização multiobjetivo, Multiobjective Evolutionary Algorithms (MOEA), Câmara térmica.

\section{INTRODUÇÃO}

Obter a representação matemática de processos (dinâmicos ou estáticos) é atividade rotineira para cientistas e estudiosos. A modelagem matemática é imprescindível na solução de problemas reais de engenharia e em diversas áreas do conhecimento.

Quando pouca ou nenhuma informação do sistema estudado é conhecida, ao processo de obtenção do modelo dáse o nome de Identificação de Sistemas (Aguirre, 2007). Os problemas abordados na identificação de sistemas podem ser simples, como o caso dos lineares, ou extremamente complexos (sistemas não lineares). Cada vez mais, os problemas aumentam em complexidade e assim, torna-

\footnotetext{
* Os autores agradecem as agências Coordenação de Aperfeiçoamento de Pessoal de Nível Superior (CAPES), Conselho Nacional de Desenvolvimento Científico e Tecnológico (CNPq) e a Universidade Federal de Ouro Preto (Número do Processo 23109.003268/2017-47) pelo apoio financeiro.
}

se necessário apresentar novos modelos que descrevam de forma mais acurada possível o sistema em estudo.

Uma representação que vem sendo abordada nas últimas décadas é a que utiliza a Série de Volterra (Aguirre, 2007; Braga, 2011; da Rosa et al., 2006). Esse tipo de modelo é interessante devido ao fato de ser híbrido, ou seja, sua estrutura pode apresentar, dependendo do sistema a ser identificado, uma parcela linear e outra não linear. Entretanto, dada a não recursão do sinal de saída do modelo, a série de Volterra, em geral, apresenta um elevado número de parâmetros para descrever ou representar o sistema, sobretudo quando o comportamento não linear do sistema depende fortemente do sinal de saída. Uma estratégia para contornar tal desvantagem envolve utilizar as funções de base ortonormal (FBO), as quais são geralmente aplicadas em modelos conhecidos (como Séries de Volterra, TS, RBF) (Campello et al., 2007a) com o intuito de, no caso das séries de Volterra, por exemplo, reduzir o número de termos necessários para a representação do sistema (Braga 
et al., 2011). Dessa forma, no presente trabalho, é utilizada a representação FBO-Volterra (Braga et al., 2011; da Rosa et al., 2006; Campello et al., 2007a).

Os modelos de Volterra são parametrizados por funções multidimensionais, os kernels. A representação FBOVolterra resume-se a projetar a base ortonormal ótima de modo a reduzir ao máximo o número de termos para a representação. Assim, escolhe-se a base ortonormal e determina-se os polos que a caracterizam. As bases discretas mais utilizadas na literatura são Laguerre, Kautz e as bases de funções generalizadas (GOBF - Generalized Orthonormal Basis Functions) (Campello e Oliveira, 2007; Campello et al., 2007a,b). Neste trabalho, optou-se por utilizar as funções de Laguerre devido à sua simplicidade, uma vez que são parametrizadas apenas por polos reais (Bokor e Schipp, 1998; Chen e Ljung, 2015).

O objetivo deste trabalho é obter os polos ótimos da base de Laguerre e definir a estrutura (o número de funções na base ortonormal) por meio de técnica meta-heurística de otimização multiobjetivo. O problema em questão pode ser classificado como multiobjetivo devido ao fato de que, além de buscar os polos ótimos que parametrizam a função ortonormal, procura-se obter uma representação que seja mais simplificada possível, ou seja, aquela que apresente o menor número possível de funções na base ortonormal.

Quando um problema envolve a minimização simultânea de um conjunto de objetivos, com a necessidade de satisfazer algumas restrições, esse pode ser caracterizado como um problema multiobjetivo (Pantuza, 2011). Algoritmos de computação evolutiva são comumente utilizados para a otimização multiobjetivo. O mais conhecido deles é o Algoritmo Genético (Srinivas e Deb, 1994; Deb et al., 2002; Boussaïd et al., 2013), o qual é utilizado no presente trabalho.

Para efeito de exemplificação, as técnicas abordadas são aplicadas na identificação do sistema de uma câmara termoeletricamente controlada que utiliza placas de Peltier, que são normalmente mais ecologicamente corretas do que os sistemas de resfriamento convencionais, para atuar no aquecimento/resfriamento da caixa.

A estrutura do trabalho é seccionada como segue. Na Seção 2 , descrevem-se brevemente o projeto e a implementação do sistema termoelétrico desenvolvido. São abordados assuntos pertinentes à identificação de sistemas de modo geral, além de focar-se nos modelos FBO-Volterra e suas características singulares, na Seção 3. A Seção 4 é destinada às técnicas de otimização utilizadas e adaptação ao problema proposto. Na Seção 5, apresentam-se os resultados obtidos e a análise de desempenho da metodologia dissertada. Finalmente, conclui-se o trabalho e sugerem-se trabalhos futuros na Seção 6.

\section{CÂMARA TERMOELETRICAMENTE CONTROLADA}

O projeto da câmara de resfriamento/aquecimento teve como objetivos:

- Elaborar e implementar um sistema de resfriamento e aquecimento por efeito Peltier, ou seja, utilizado módulos TEC (Thermoelectric Colling);
- Elaborar e implementar um sistema de potência capaz de atuar os módulos TEC de forma reversível e eficiente.

Sistemas de refrigeração por efeito Peltier, apesar de apresentarem desempenho inferior, são mais amigáveis ao meio ambiente que sistemas convencionais (refrigeradores). Sistemas que utilizam elementos termoelétricos são amplamente estudados por diversos autores, como em Zhang (2013) em que um sistema de controle de temperatura para uma câmara de testes para diodos laser é proposto, ou em Riffat et al. (2001) em que um sistema de refrigeração de uma caixa é proposto e é mostrado que dissipadores de calor com aletas são recomendados para esse tipo de sistema.

Sistemas térmicos são interessantes no presente trabalho por comumente apresentarem dinâmica não linear, sendo, portanto, um bom exemplo para avaliar o desempenho da técnica de identificação associada à representação FBOVolterra para sistemas não lineares.

\subsection{Características da Câmara}

A câmara tem $40 \mathrm{~cm}$ de comprimento, $30 \mathrm{~cm}$ de largura e $30 \mathrm{~cm}$ de altura. Dois orifícios quadrados de $100 \mathrm{~cm}^{2}$ foram abertos para acomodar duas estruturas, cada uma contendo um módulo Peltier TEC 12715, dois dissipadores de calor além de duas ventoinhas. O módulo Peltier é posicionado entre os dois dissipadores de calor, que são responsáveis por aumentar a área superficial do módulo Peltier e, assim, facilitar a transferência de calor. Devido à dificuldade de inserção dos conjuntos de módulos Peltier, dissipadores e ventoinhas nos orifícios laterais, sobraram pequenos vãos que poderiam interferir na eficiência do sistema. Para contornar esse problema, preencheram-se os espaços vazios com pedaços de isopor. A função das ventoinhas internas é de espalhar o calor uniformemente dentro da câmara e as ventoinhas externas facilitar a troca de calor com o meio externo. Em Pereira et al. (2018) pode-se visualizar uma fotografia tirada da parte externa da câmara.

\subsection{Sistema de Acionamento}

Como dito previamente, fez-se necessária a utilização de dois módulos Peltier TEC 12715. Uma dificuldade encontrada no projeto foi a determinação de como realizar a ligação dos módulos Peltier. Uma possibilidade seria utilizar duas fontes de alimentação distintas para cada módulo, o que ajudaria quando fossem aplicadas técnicas de controle. Outra maneira de acioná-las, seria utilizar uma única fonte de alimentação com as placas conectadas em paralelo. E, por último, realizar a conexão dos módulos em série com uma única fonte de alimentação. A primeira opção foi descartada por questões orçamentárias e de eficiência energética. Como apresentado em Lugão (2016), conectar placas Peltier em série eletricamente é a opção mais adequada.

Sendo assim, os módulos TEC foram conectados em série e alimentados por um circuito de potência Ponte $\mathrm{H}$ de MOSFETs para que pudessem ser usados para resfriar ou aquecer a câmara. 


\section{MODELAGEM DO SISTEMA}

Prever séries temporais (previsão do tempo, de mercado, de demanda energética, etc.), estudar o comportamento de sistemas e realizar o controle são aplicações comuns para a identificação de sistemas.

Várias são as formas de se representar sistemas dinâmicos. Umas das estruturas mais conhecidas e utilizadas é a (N)ARX - (Nonlinear) Auto-Regressive with eXogenous inputs — em que a saída do sistema em um determinado instante de tempo discreto é representada utilizando amostras passadas dos seus sinais de entrada e saída (E/S) (Campello et al., 2007a; Aguirre, 2007). Também são discutidas as representações Volterra, fuzzy, neurais, NARMAX nas bibliografias mais conceituadas da área (Campello et al., 2007a).

\subsection{Modelos de Volterra}

Visto como uma generalização do modelo FIR (Finite Impulse Response), as séries de Volterra são um tipo de representação polinomial sem realimentação do sinal de saída, o que permite contornar o problema de realimentação do erro. A ausência de realimentação dos regressores de saída proporciona a não polarização do estimador de mínimos quadrados (Aguirre, 2007). Essa representação é descrita por

$$
\begin{gathered}
y(k)=h_{0}+\sum_{k_{1}=0}^{\infty} h_{1}\left(k_{1}\right) u\left(k-k_{1}\right)+ \\
\sum_{k_{1}=0}^{\infty} \sum_{k_{2}=0}^{\infty} h_{2}\left(k_{1}, k_{2}\right) u\left(k-k_{1}\right) u\left(k-k_{2}\right)+\cdots+ \\
\sum_{k_{1}=0}^{\infty} \ldots \sum_{k_{m}=0}^{\infty} h_{m}\left(k_{1}, \cdots, k_{m}\right) u\left(k-k_{1}\right) \cdots u\left(k-k_{m}\right)+\ldots
\end{gathered}
$$

em que $u(k), \ldots, u\left(k-k_{m}\right)$ é o sinal de entrada e $h(k), \ldots, h\left(k_{1}, \ldots, k_{m}\right)$ são os kernels da série de Volterra.

Uma vantagem de sua utilização é que não é preciso apontar explícita e previamente a ordem do processo (Braga, 2011). Além disso, esse modelo tem como característica a hibridização e pode, portanto, apresentar um termo linear e um não linear na representação de sistemas. Por outro lado, a representação por modelos de Volterra geralmente apresentam a desvantagem de necessitar de um grande número de termos na sua estrutura. É fácil perceber esse empecilho ao observar a expressão em (1), em que o sinal de entrada $u(k), \ldots, u\left(k-k_{m}\right)$ é mapeado não linearmente com os kernels $h(k), \ldots, h\left(k_{1}, \ldots, k_{m}\right)$ da série de Volterra para expressar a saída estimada do sistema.

Os kernels da série de Volterra possuem propriedades que são importantes na modelagem matemática dos sistemas. Uma delas é a causalidade, propriedade essa que é facilmente observada em (1). Outra propriedade interessante é a fading memory, que permite o truncamento da série de Volterra sem que haja perda significativa das informações relevantes do sistema. Essa propriedade vem do fato de que respostas a qualquer entrada desaparecem com o passar do tempo (Eykhoff, 1974). A última propriedade interessante é a que permite a simetrização dos kernels. Essa propriedade reduz o custo computacional, uma vez que o número de termos a serem estimados pode reduzir consideravelmente. Sugere-se a leitura de Rugh (1981), no qual, em um procedimento detalhado, é apresentada essa propriedade.

Apesar da propriedade de fading memory permitir o truncamento da série de Volterra, como apresentado no parágrafo anterior, a representação ainda pode necessitar de um número elevado de termos. A solução encontrada é utilizar as funções de base ortonormal em conjunto com a série.

Os kernels podem ser desenvolvidos em FBOs desde que sejam estáveis. Se, hipoteticamente, $h_{m}\left(k_{1}, k_{2}, \ldots, k_{m}\right)=$ $0, \forall k_{i}>\epsilon_{m}$, então (1) pode ser reescrita como (Campello et al., 2007a)

$$
y(k)=\sum_{m=1}^{M} \sum_{k_{1}}^{\epsilon_{m}} \cdots \sum_{k_{m}}^{\epsilon_{m}} h_{m}\left(k_{1}, \ldots, k_{m}\right) \prod_{j=1}^{m} u\left(k-k_{j}\right) .
$$

Assim, desenvolvendo-se todos os kernels em uma única base de funções, o desenvolvimento $m$-dimensional do kernel de grau $m$ é

$$
h\left(k_{1}, \ldots, k_{m}\right)=\sum_{i_{i}}^{\infty} \cdots \sum_{i_{m}}^{\infty} c_{i_{1}, \ldots, i_{m}} \prod_{j=1}^{m} \phi_{i_{j}}\left(k_{j}\right),
$$

sendo $c_{i_{1}, \ldots, i_{m}}$, os parâmetros a serem estimados, e $\phi_{i_{j}}$, a base de funções ortonormais utilizadas. Substituindo (3) em (2), tem-se o modelo FBO-Volterra, dado por

$$
y(k)=\sum_{m=1}^{M} \sum_{i_{1}}^{\infty} \cdots \sum_{i_{m}=1}^{\infty} c_{i_{1}, \ldots, i_{m}} \prod_{j=1}^{m} l_{i_{j}}(k),
$$

$\operatorname{com} l_{i_{j}}(k)$ sendo a saída do i-ésimo filtro ortonormal, dado por

$$
l_{i_{j}}=\sum_{\tau=0}^{\infty} \phi(\tau) u(k-\tau),
$$

em que $\phi(\tau)$ é a função ortonormal no domínio do tempo. Por simplicidade, a função ortonormal utilizada neste trabalho foi a de Laguerre, que no domínio $z$ é expressa por (Campello et al., 2007a)

$$
\Phi_{i}(z)=\frac{\sqrt{1-p^{2}}}{z-p}\left(\frac{1-p z}{z-p}\right)^{i-1}
$$

em que $i=1,2, \ldots$ e $p \in\{p \in \mathbb{R}|| p \mid<1\}$ é o polo estável que parametriza as FBO.

Método dos Mínimos Quadrados. Tratando cada elemento dos kernels da série de Volterra como um parâmetro independente a ser estimado, o modelo de Volterra torna-se linear nos parâmetros e, assim, podem-se aplicar métodos de identificação clássicos, como o método dos mínimos quadrados.

Realizando o mapeamento não linear descrito em (4), temse que as linhas da matriz de regressores $\Lambda$ são dadas por

$$
\begin{aligned}
& \Lambda=\left[\begin{array}{llll}
1 & l_{1}(k) & \cdots & l_{m_{1}}(k) \\
l_{1}(k) l_{1}(k)
\end{array}\right. \\
& \left.l_{1}(k) l_{2}(k) \cdots l_{m_{2}-1} l_{m_{2}} l_{m_{2}} l_{m_{2}} \cdots\right] \text {. }
\end{aligned}
$$

Além disso, os parâmetros a serem estimados são representados pelo vetor de parâmetros

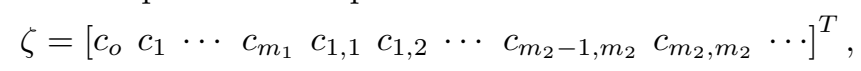

os quais podem ser obtidos fazendo

$$
\zeta=\left(\Lambda^{T} \Lambda\right)^{-1} \Lambda^{T} y
$$


com y sendo a saída real do sistema.

\section{OTIMIZAÇÃO}

Em variados casos do cotidiano, encontram-se problemas com múltiplos objetivos na área de otimização. O objetivo da otimização multiobjetiva é alcançar um grupo de soluções ótimas que contemplem uma gama de metas obedecendo a um grupo de restrições (Pantuza, 2011). Do grupo de resultados encontrados é de responsabilidade do engenheiro/pesquisador elencar a decisão de acordo com o problema pertencente.

Usa-se aqui o trabalho de Pantuza (2011), o qual discorre que um problema de otimização multiobjetivo manifestase da seguinte maneira

$$
\begin{gathered}
\min z=f(x)=\left(f_{1}(x), f_{2}(x), \cdots, f_{O B}(x)\right) \\
\text { s a } g(x)=\left(g_{1}(x), g_{2}(x), \cdots, g_{r}(x)\right) \leq b \\
x=\left(x_{1}, x_{2}, \cdots, x_{n}\right) \in \mathcal{X} \\
z=\left(z_{1}, z_{2}, \cdots, z_{n}\right) \in \mathcal{Z}
\end{gathered}
$$

em que $x$ é o vetor de decisão, $O B$ é o número de objetivos, $r$ é o número de restrições, $z$ é o vetor objetivo e $\mathcal{X}$ é o espaço de busca de decisões.

O grupo de soluções ótimas recebe o nome Pareto-ótimo. Um vetor $z$ pertence ao grupo de Pareto-ótimo caso não haja nenhum vetor $z$ que possa progredir algum objetivo, mas sem causar piora em ao menos um objetivo (Pantuza, 2011). Assim, quando a solução é pior que a outra em todos os objetivos, ela encontra-se dominada por essa outra.

\subsection{Algoritmo Genético Multiobjetivo}

O Algoritmo Genético é uma das meta-heurísticas mais habitualmente utilizadas em problemas de otimização multiobjetivo. Essa forma de algoritmo faz parte da classe dos MOEA (Multiobjective Evolutionary Algorithms) (Deb et al., 2002). Pode-se encontrar em Pantuza (2011), um breve histórico do desenvolvimento dessa classe de algoritmo.

NSGA-II. O NSGA-II (Nondominated Sorting Genetic Algorithm II), foi o algoritmo multiobjetivo escolhido para aplicação neste trabalho. Essa escolha se dá, especialmente, por sua acessível implementação. O NSGA-II foi proposto por Deb et al. (2002) como uma melhora do NSGA (Srinivas e Deb, 1994).

Refere-se a um algoritmo fundado em uma ordenação elitista por dominância, que visa categorizar os indivíduos de um grupo em níveis relacionados ao seu grau de dominância (Pantuza, 2011). No Algoritmo 1, expõe-se o pseudocódigo do algoritmo Fast Non-dominanted Sort, que é responsável por decretar as soluções dentro da população em diferentes frentes de Pareto.

Outra circunspeção utilizada pelo NSGA-II é a ponderação da distância de uma solução para outra dentro de uma mesma Frente de Pareto, para assegurar a diversidade da frente de Pareto (Deb et al., 2002). No Algoritmo 2, apresenta-se o pseudocódigo do algoritmo crowding distance, que é incumbido pelo cálculo da distância entre duas soluções dentro da mesma frente de Pareto (buscase sempre maximizar a distância, de modo a atestar uma maior diversidade dentro da Frente de Pareto).
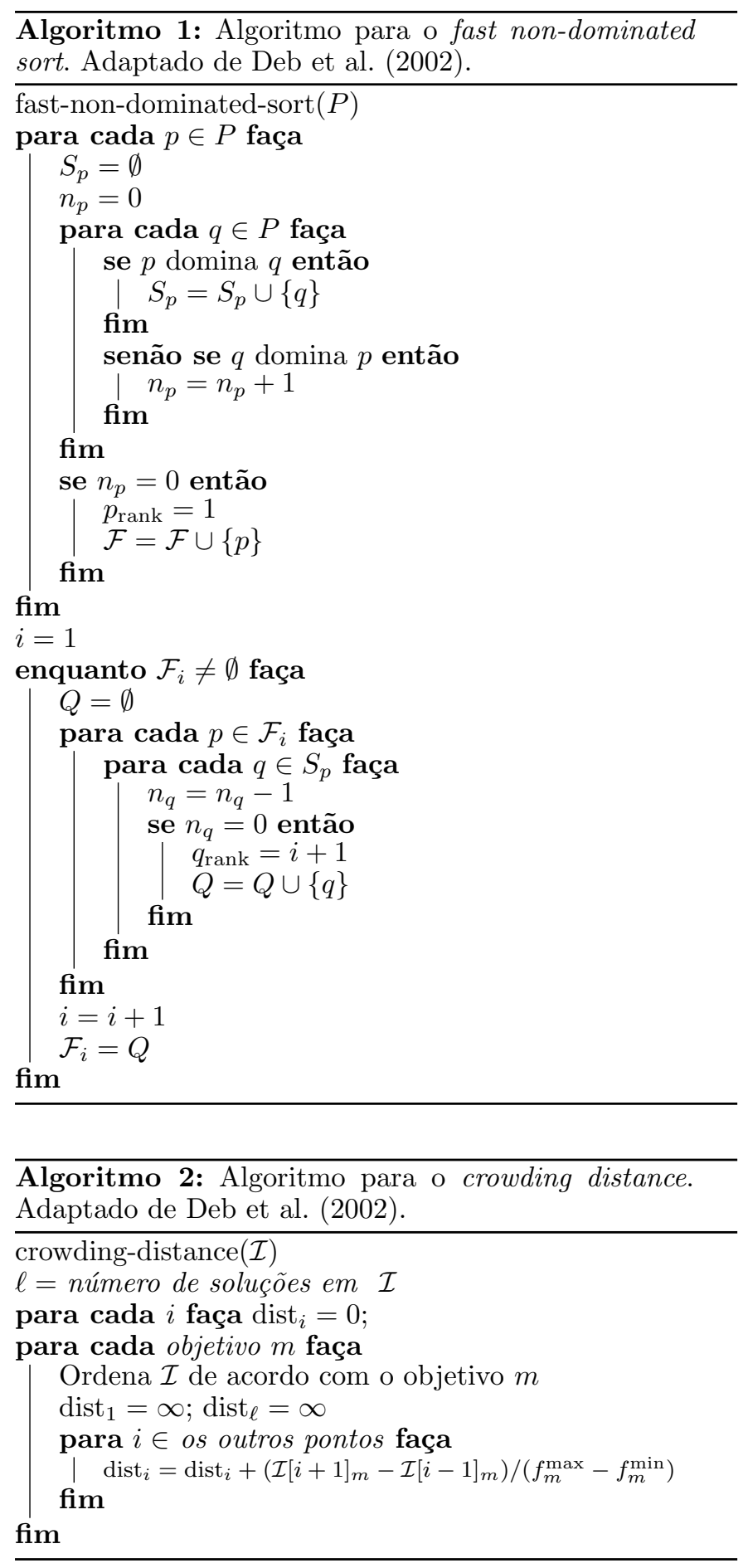

\section{RESULTADOS E DISCUSSÕES}

O sistema usado como exemplo para a identificação neste trabalho é o apresentado na Seção 2. Com uma amostragem de $0,8 \mathrm{~s}$, decimaram-se os dados em intervalos de $8 \mathrm{~s}$ (ou 10 amostras). O valor de tensão, sinal de entrada $u$, aplicado nas placas é um sinal que varia de -12 a $12 \mathrm{~V}$ e o sinal de saída $y$, temperatura no interior da câmara.

Para o processo de identificação, definiu-se uma estrutura não linear, composta por um kernel de primeira ordem e um kernel de segunda ordem com funções de Laguerre. Os objetivos do algoritmo de otimização foram: (i) minimizar 


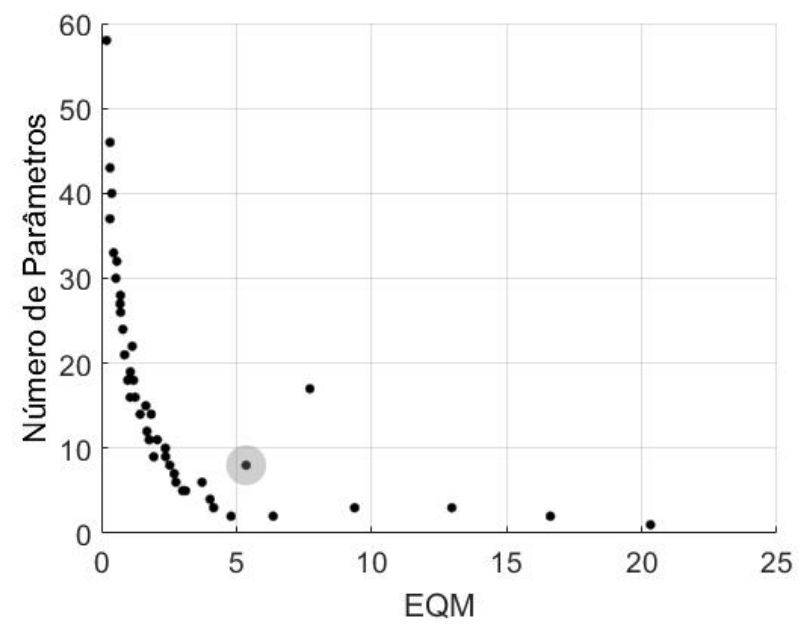

Figura 1. Pareto-ótimo obtido pelo algoritmo NSGAII no processo de identificação do modelo da caixa termoeletricamente controlada.

o Erro Quadrático Médio (EQM) entre o sistema e o modelo e; (ii) reduzir o número de parâmetros do modelo.

O Algoritmo Genético (AG) é desenvolvido com indivíduos representados por um vetor contendo os polos da FBO, sendo um polo para o kernel de primeira ordem e dois polos iguais (simetrizados) para o kernel de segunda ordem. Além disso, o vetor de indivíduos possui o número de funções $n_{1}$ e $n_{2}$. Os parâmetros utilizados no AG são: tamanho da população (50 indivíduos), número de gerações (200) e método de seleção dos pais (torneio de 3 elementos), levando em conta que essa é uma configuração que apresentou os melhores resultados com um custo computacional aceitável, cerca de 1 minuto e 46 segundos em um computador com alta capacidade de processamento.

O algoritmo NSGA-II obteve o Pareto-ótimo apresentado na Figura 1. Na Tabela 1 também pode-se observar o Pareto-ótimo, na qual expõem-se as combinações ótimas obtidas, sendo $n_{1}$ e $n_{2}$, o número de funções utilizadas, $\mathrm{Polo}_{1}$ e $\mathrm{Polo}_{2}$, os polos empregados para parametrizar, respectivamente, os kernels de primeira e segunda ordens, $E Q M$, o erro quadrático médio obtido pelo procedimento de identificação, $E Q M_{\mathrm{V}}$, o erro quadrático médio obtido na etapa de validação do modelo, e $N_{p}$, o número total de parâmetros identificados.

No processo de otimização, a escolha da melhor estrutura é feita considerando-se o EQM. No entanto, na tomada de decisão (posterior à otimização) deve-se evitar soluções do Pareto Ótimo cujo modelo é sobreparametrizado (possuem um EQM de validação elevado).

Levando isso em conta, um dos melhores modelos obtido foi o simétrico (polos iguais), com 4 funções em $n_{1}$, com Polo $_{1}=0,8813$, e 2 funções em $n_{2}$, com $\mathrm{Polo}_{2}=0,8635$, totalizando 8 parâmetros, destacado na Tabela 1 e na Figura 1 . Na Figura 2, apresenta-se a saída estimada do modelo escolhido. Também é possível avaliar o desempenho do método por meio da Figura 3, na qual é mostrada a saída para dados de validação usando o primeiro modelo citado.
Tabela 1. Resultados ótimos obtidos pelo algoritmo NSGA-II no processo de identificação do modelo da caixa termoeletricamente controlada. A linha em destaque evidencia o melhor resultado escolhido.

\begin{tabular}{cccccrc}
\hline \hline$n_{1}$ & $n_{2}$ & Polo $_{1}$ & Polo $_{2}$ & $E Q M$ & $E Q M_{\mathrm{V}}$ & $N_{p}$ \\
\hline \hline 1 & 0 & 0.9750 & 0.9870 & 6.362 & 7.883 & 2 \\
2 & 0 & 0.8901 & 0.8709 & 9.375 & 13.380 & 3 \\
2 & 1 & 0.9651 & 0.8907 & 4.025 & 14.034 & 4 \\
4 & 0 & 0.9965 & 0.9679 & 3.011 & 21.531 & 5 \\
2 & 2 & 0.9647 & 0.8720 & 3.727 & 13.748 & 6 \\
4 & 1 & 0.9714 & 0.9995 & 2.762 & 17.730 & 6 \\
3 & 2 & 0.9611 & 0.9996 & 2.694 & 17.627 & 7 \\
$\mathbf{4}$ & $\mathbf{2}$ & $\mathbf{0 . 8 8 1 3}$ & $\mathbf{0 . 8 6 3 5}$ & $\mathbf{5 . 3 6 0}$ & $\mathbf{7 . 3 9 7}$ & $\mathbf{8}$ \\
5 & 2 & 0.9724 & 0.9992 & 2.378 & 19.124 & 9 \\
2 & 3 & 0.9635 & 0.9975 & 1.942 & 49.739 & 9 \\
6 & 2 & 0.9971 & 0.9982 & 2.368 & 24.605 & 10 \\
4 & 3 & 0.9502 & 0.9976 & 1.771 & 44.215 & 11 \\
5 & 3 & 0.9805 & 0.9981 & 1.688 & 42.969 & 12 \\
3 & 4 & 0.9358 & 0.9973 & 1.847 & 38.956 & 14 \\
4 & 4 & 0.9703 & 0.9966 & 1.647 & 62.066 & 15 \\
5 & 4 & 0.9170 & 0.9991 & 1.250 & 50.808 & 16 \\
8 & 4 & 0.9739 & 0.9979 & 1.073 & 83.032 & 19 \\
10 & 6 & 0.9660 & 0.9951 & 0.572 & 82.25 & 32 \\
\hline \hline & & & & & & \\
\hline
\end{tabular}

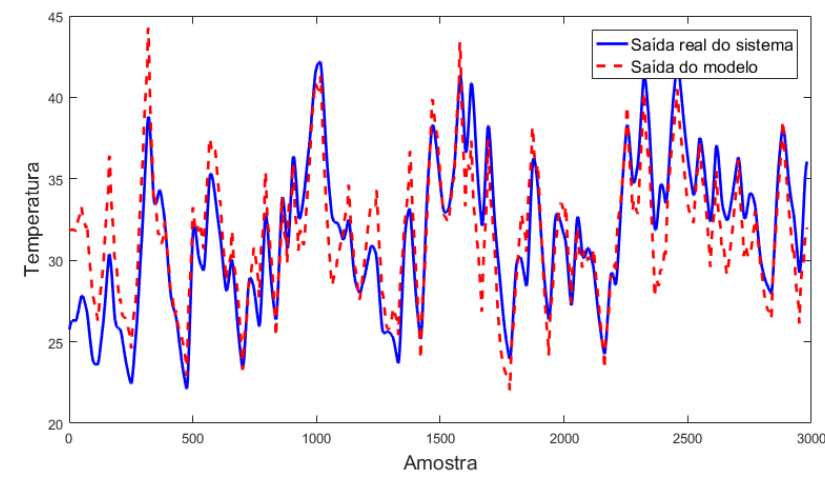

Figura 2. Resultados da estimação obtidos com $n_{1}=4$, $n_{2}=2$ e Polo $_{1}=0,8813$ e Polo $_{2}=0,8635$ : Saída real do sistema (linha azul contínua) e Saída do modelo (linha vermelha tracejada).

Uma forma encontrada para verificar a eficácia do método foi desenvolver um modelo mais simples e compará-lo aos obtidos nesse trabalho (Tabela 1 ). No caso, optou-se por um modelo ARX, um dos mais conhecidos daqueles modelos tradicionais. Após algumas verificações, o melhor modelo ARX obtido é

$$
\begin{aligned}
& y(k)=a_{1} y(k-1)+a_{2} y(k-2)+a_{3} y(k-3)+ \\
& \quad+b_{1} u(k-1)+b_{2} u(k-2)+b_{3} u(k-3),
\end{aligned}
$$

em que $u(k)$ é a entrada do sistema, $y(k)$ a saída, e $a_{(\cdot)}$ e $b_{(\cdot)}$ os termos a serem determinados por meio de mínimos quadrados. Esse modelo apresentou um EQM de 2.875 e um $E Q M_{V}$ de 21.427. Fica claro que, devido à realimentação, o $E Q M$ do modelo ARX se equipara ao $E Q M$ dos modelos obtidos pela representação FBO-Volterra. No entanto, quando analisa-se o $E Q M_{V}$ é notória a superioridade do método aqui desenvolvido. Uma explicação razoável para a inferioridade no desempenho dos modelos 


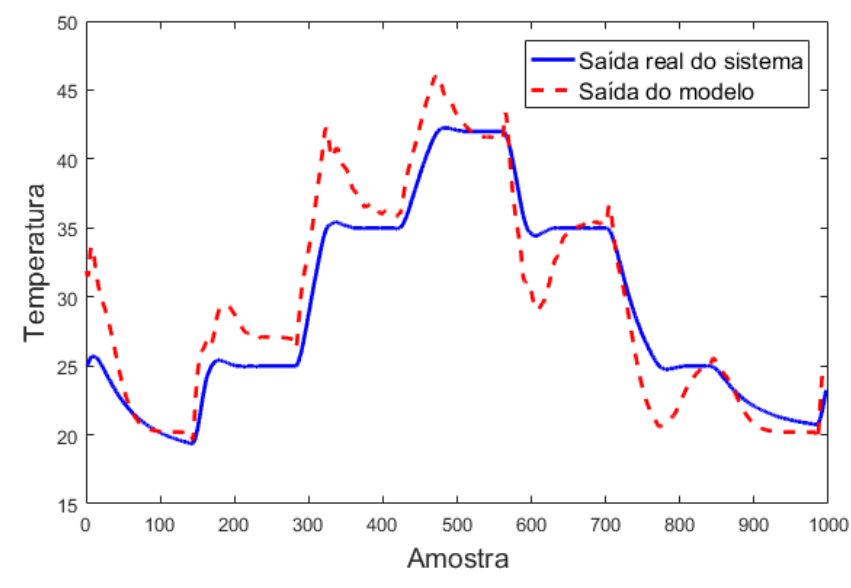

Figura 3. Validação do modelo $\left(n_{1}=4, n_{2}=2\right.$ e Polo $_{1}=$

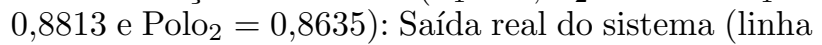
azul contínua) e Saída do modelo (linha vermelha tracejada).

ARX desenvolvidos é a existência de realimentação do sinal de saída, que diminui a capacidade de generalização do modelo.

\section{CONCLUSÕES}

Modelar sistemas não lineares por meio da representação FBO-Volterra é uma técnica que se mostra satisfatória, principalmente pelo fato das representações terem a característica de serem do tipo "malha aberta". Dessa forma, o erro da saída não é realimentado à entrada. O problema da grande quantidade de parâmetros é contornado usando as funções de bases ortonormais. Escolher o conjunto de polos adequado que parametrize as $\mathrm{FBO}$ faz com que o número de termos a serem determinados seja reduzido.

Uma forma de alcançar esse objetivo é por empregar o método de otimização multiobjetivo NSGA-II, como mostrado no presente trabalho, para a identificação do modelo de uma câmara termoeletricamente controlada. A otimização multiobjetivo se mostrou uma ferramenta importante e poderosa, que pode ser aplicada em diversos problemas da engenharia e outras áreas de pesquisa.

Como trabalhos futuros, espera-se empregar estruturas mais complexas na série de Volterra, como kernels de terceira ordem, assim como bases de funções ortonormais mais complexas, como as de Kautz e generalizadas.

\section{REFERÊNCIAS}

Aguirre, L.A. (2007). Introdução à Identificação de Sistemas: técnicas lineares e não-lineares aplicadas a sistemas reais. Editora UFMG.

Bokor, J. e Schipp, F. (1998). Approximate identification in Laguerre and Kautz basis. Automatica, 34(4), 463468.

Boussaïd, I., Lepagnot, J., e Siarry, P. (2013). A survey on optimization metaheuristics. Information Sciences, 237, 82-117.

Braga, M.F., Machado, J.B., Campello, R.J.G.B., e do Amaral, W.C. (2011). Optimization of Volterra models with asymmetrical kernels based on generalized orthonormal functions. In The IEEE 19th Mediterranean Conference on Control \& Automation (MED), 1052-1058.

Braga, M.F. (2011). Modelos de Volterra: Identificação Não Paramétrica e Robusta Utilizando Funções Ortonormais de Kautz e Generalizadas. Dissertação de Mestrado, Universidade de Campinas.

Campello, R.J.G.B. e Oliveira, G.H.C. (2007). Modelos não-lineares. In L.A. Aguirre, A.P.A. Silva, M.F.M. Campos, e W.C. Amaral (eds.), Enciclopédia de Automática, volume 3. Edgar Blücher.

Campello, R.J.G.B., Oliveira, G.H.C., e Amaral, W.C. (2007a). Identificação e controle de processos via desenvolvimentos em série ortonormais: Parte a: Identificação. Controle \& Automação, 18(3), 301-321.

Campello, R.J.G.B., Oliveira, G.H.C., e Amaral, W.C. (2007b). Identificação e controle de processos via desenvolvimentos em série ortonormais: Parte b: Controle. Controle \& Automação, 18(3), 321-336.

Chen, T. e Ljung, L. (2015). Regularized system identification using orthonormal basis functions. In 2015 European Control Conference (ECC), 1291-1296.

da Rosa, A., Amaral, W.C., e Campello, R.J.G.B. (2006). Desenvolvimento de modelos de Volterra usando funções de Kautz e sua aplicação à modelagem de um sistema de levitação magnética. In Anais do Congresso Brasileiro de Automática, 274-279.

Deb, K., Pratap, A., Agarwal, S., e Meyarivan, T. (2002). A fast and elitist multiobjective genetic algorithm: NSGA-II. IEEE Transactions on Evolutionary Computation, 6(2), 182-197.

Eykhoff, P. (1974). System identification, 197. JW Arrowsmith Ltd, Bristol, Great Britain.

Lugão, J.R.L. (2016). Controlador de temperatura para célula de medição de propriedades de líquidos por ultrassom. Dissertação de Mestrado, Universidade Estadual Paulista (UNESP).

Pantuza, G. (2011). Métodos de otimização multiobjetivo e de simulação aplicados ao problema de planejamento operacional de lavra em minas a céu aberto. Dissertação de Mestrado, Programa de Pós-Graduação em Engenharia Mineral - PPGEM da Universidade Federal de Ouro Preto, Ouro Preto.

Pereira, A., Vianna, L., Keles, N., e Campos, V. (2018). Tensor product model transformation simplification of takagi-sugeno control and estimation laws - An application to a thermoelectric controlled chamber. Acta Polytechnica Hungarica, 15, 13-29.

Riffat, S., Omer, S., e Ma, X. (2001). A novel thermoelectric refrigeration system employing heat pipes and a phase change material: an experimental investigation. Renewable Energy, 23(2), 313-323.

Rugh, W.J. (1981). Nonlinear Systems Theory - The Volterra/Wiener Approach. The Johns Hopkins University Press, Baltimore.

Srinivas, N. e Deb, K. (1994). Multiobjective optimization using nondominated sorting in genetic algorithms. Evolutionary computation, 2(3), 221-248.

Zhang, S.N. (2013). Thermoelectric Cooler Based Temperature Controlled Environment Chamber Design for Application in Optical Systems. Tese de Doutorado, Virginia Tech. 